\title{
ОБ ИЗМЕНЕНИИ ОБЬЕМНОГО ВЕСА, ПРОЧНОСТИ И МОДУЛЯ УПРУГОСТИ ГАЗОКУКЕРМИТА В БЛОКАХ, ЗАЛИТЫХ В ВЕРТИКАЛЬНЫХ ФОРМАХ
}

\author{
В. РАЯДНА, \\ кандидат технических наук
}

Х. ЭЭСОРГ

\begin{abstract}
Свойства газокукермита * в стронтельных деталях, залитых в вертикальных формах в случае значительного их размера в направлении заливки, могут сушественно изменяться по высоте заливки. Кроме того, в процессе изготовлення блоков газокукермнт значительно уплотняется у стенок формы, что также может быть причнной изменения его прочности и деформативных свойств в деталях. Поэтому в газокукер митовых деталях, залитых в вертикальных формах, распределение величин объемного веса, прочности н модуля упругости материала блока может нметь достаточно сложную картину.
\end{abstract}

В 1962 г. в Институте стронтельства и строительных матерналов проводнлось нсследование прочностных и деформативных свойств газокукермитовых блоков, зал:тых в вертикальных формах. Блокн были изготовлены на Ахтмеском комбинате стронтельных материалов. Размеры нспытанных блоков представлены на рнс. 1. Для опре-деления объемного веса и прочности на сжатие материала блоков, из них высверливались цилиндры $\varnothing=h=5$ сн, которые испытывались в высушенном состоянин. Места высверливания цилиндров показаны на рис. 1.

Нзменение объемного веса $\delta \gamma$, прочности $\delta R_{ц}$ н модуля упругостн $\delta H$ на однн погонный метр длины в направленин заливкн блока определились по формулам:

$$
\delta \gamma=\frac{\gamma_{\mathrm{H}}-\gamma_{\mathrm{B}}}{c} ; \delta R_{\mathrm{L}}={\frac{R_{\mathrm{H}}-R_{\mathrm{B}}}{c}}^{c} ; \delta H=\frac{H_{\mathrm{H}}-H_{\mathrm{B}}}{c}
$$

Fne

$Y_{\mathrm{B}}, R_{\mathrm{B}}, H_{\mathrm{B}}$ - средние значення объемного веса, прочности на сжатие н модуля упругости цилиндров, высверленных в точках 1 и 2 (см. рис. 1);

$\gamma_{\mathrm{H}}, R_{\mathrm{H}}, H_{\mathrm{H}}$ - средние значения объемного веса, прочности на сжатне и модуля. упругости цилиндров, высверленных в точках 4 н 5 (см. рнс. 1);

c - расстояние между точкамн 1 и 4 или 2 и 5 в направлении заливки.

На основании испытаний 40 газокукермитовых блоков, с объемным весом $800 \div 1100 \mathrm{\kappa c} / \mathrm{m}^{3}$, средние значения изменения объемного веса и прочности на сжатие

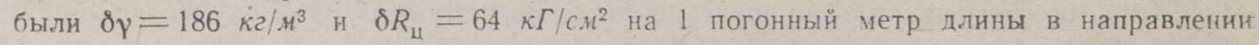
заливкн блока.

* Газокукермитом называется газобетон на сланцезольном вғжушем. 
Для детального нсследования неоднородностн газокукермнта в пределах блока высверливалнсь нз одного блока пнлиндры, днаметром 5 сл, длиной больше 15 см, шатом 20 см в направленни длнны (направленне z на рнс. 1) н шагом 6 см в направленин шнрнны (направленне $y$ на рис. 1). На этих щилиндрах определялнсь значення динамического модуля упругости $H$ на основании собственных поперечных колебаний образцов. После определения модуля упругости эти цнлнндры распиливались на части длнной по $5 \mathrm{~cm}$, которые, после высушивання прн температуре $105-$ $110^{\circ} \mathrm{C}$, взвешивались для определения объемного веса и испытывалнсь на сжатие.

На рнс. 2,3 н 4 изображены линии постоянных значений объемного веса $\gamma$, прочностн на сжатие $R_{\mu}$ н модуля упругости $H$. Нз рнсунков вндно, что в верхней частн блюка в направленин заливки величины $\gamma, R_{\text {ц }}$ и $H$ нзменяются сравнительно медленно, a в нижней части блока нзменение величин $\gamma, R_{ц}$ н $H$ значительно больше. $\mathrm{Y}$ торцов величнны $\gamma$. $R_{\text {מ }}$ н $H$ нмеют значнтельно большне значения, чем в сред-

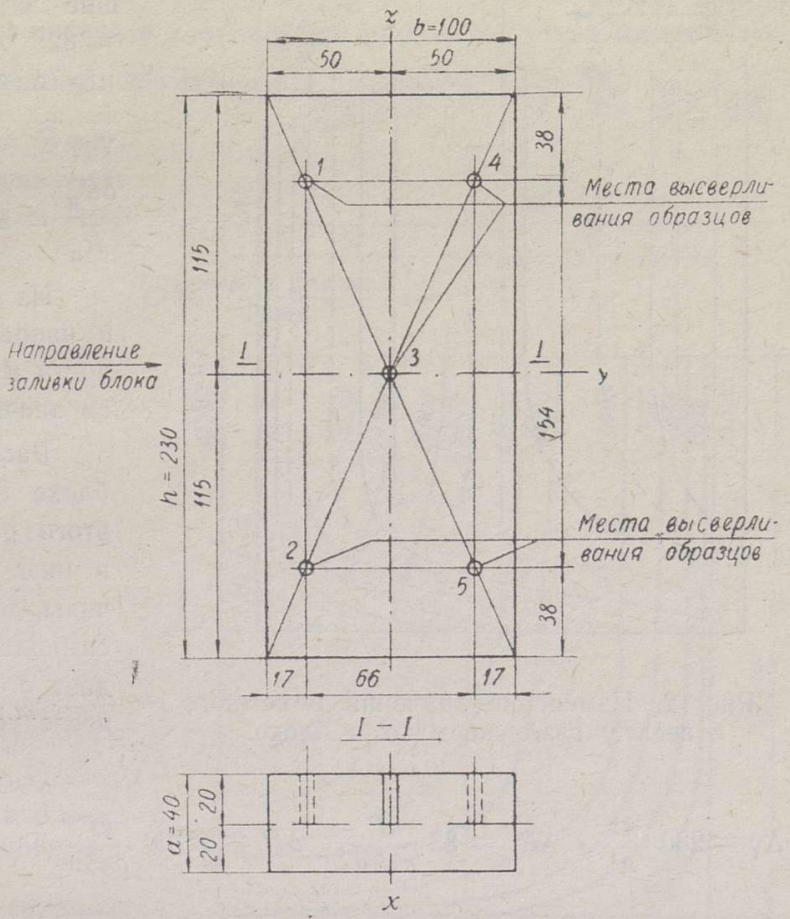

Рис. 1. Места высверливания образцов нз блока н система координат на блоке. ней частн блока. Резкое уве-

диченне этнх величин у торцов объясняется уплотнением газокукермита у стенок формы во время изготовлення блоков. В направленни толщнны блока также было заметно уплотнение газокукермита у боковых поверхностей блока.

Среднне значения в блоке объемного веса $\gamma$, прочности $R_{\text {ц }}$ и модуля упругости $H$, определенные как среднне арифметические результатов нспытания цилиндров, высверленных нз блоков согласно схеме на рис. 1 , обозначаем буквами $\bar{\gamma}^{\prime}, \bar{R}_{ц}^{\prime}$ и $\bar{H}^{\prime}$. Более точно среднне значення величин $\gamma, R_{\mu}$ н $H$ характеризуются ннтегральнымн велнчннамн $\bar{\gamma}=\frac{1}{V} \int_{V} \gamma d V, \quad \bar{R}_{ц}=\frac{1}{V} \int_{V} R_{u} d V, \quad \bar{H}=\frac{1}{V} \int_{V} H d V$, где $V=a b h$ объем блока, ннтегрнрование пронзводится по целсму объему блока. Так как нахожденне величнн $\bar{\gamma}, \vec{R}_{\text {ц }}$ и $\bar{H}$ обычно практнчески затруднено или совсем невозможно, вознн кает вопрос, как величнны $\bar{\gamma}^{\prime}, \bar{R}_{ц}^{\prime}$ и $\bar{H}^{\prime}$ характернзуют действнтельные средние значення объемного веса $\bar{\gamma}$, прочности $\vec{R}_{ц}$ и модуля упругости $\vec{H}$.

Согласно распределенню величин $\gamma, R_{ц}$ н $H$, изображенных на рнс. 2,3 н 4 , значення этнх величин являются следуюшими: $\bar{\gamma}=983 \kappa 2 / \mathrm{cm}^{3}, \bar{\gamma}^{\prime}=961 \kappa 2 / \varkappa^{3}, \vec{R}_{ц}=$ $=146 \kappa \Gamma / c \mu^{2}, \bar{R}_{ц}^{\prime}=140 \kappa \Gamma / c \mu^{2}, \bar{H}=51500 \kappa \Gamma / c \mu^{2}$ н $\bar{H}^{\prime}=49100 \kappa \Gamma / c \mu^{2}$. Следовательно, по результатам испытаннй образцов, высверленных по схеме на рис. 1, вычнсленные значения объемного веса, прочности н модуля упругости $\vec{\gamma}^{\prime}, \vec{R}_{\mathrm{u}}^{\prime}$ н $\bar{H}^{\prime}$ хорошо характернзуют действительные средние значения этих величнн $\bar{\gamma}, \bar{R}_{ц}$ и $\bar{H}$, отличаясь от последних меньше чем на $5 \%$. 


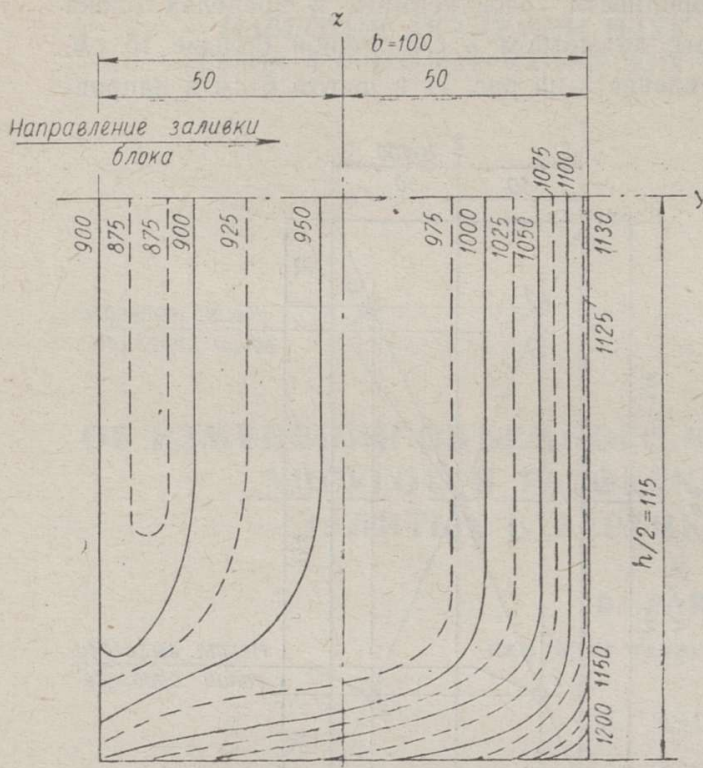

Рнс. 2. Нзменение значений объемного веса $\gamma$ газокукермита в блоке.
Используя распределение величин $\gamma, R_{\text {ц }}$ и $H$, изображенных на рнс. 2,3 и 4 , по формулам (1) имеем следую. щне значения: $\delta \gamma=187 \kappa 2 / \mu^{4}, \delta R_{\mu}=$ $=82 \kappa \Gamma / c \mu^{2} \mu, \quad \delta H=18700 \quad \kappa \Gamma / C \mu^{2} \mu$, что в процентах от средних величин $\bar{\gamma}, \bar{R}_{\Perp}$ и $\bar{H}$ составляет: $\frac{\delta \gamma}{\bar{\gamma}}=19 \%$; $\frac{\delta R_{\mathrm{u}}}{\bar{R}_{\mathrm{u}}}=56 \%$ и $\frac{\delta H}{\bar{H}}=36 \%$.

Из вышеизложенного следует, что В направлении заливки значения прочности и модуля уп́ругости увеличивают ся значительно больше объемного веса

Распределение величин $\gamma, R_{\text {ц }}$ и $H$ в блоке не является линейным; в сплу этого разннца значений величнн верха и низа заливки (обозначаем эти величнны, деленные на высоту заливки блока символами $\Delta \gamma, \Delta R_{\text {ц }}$ и $\left.\Delta H\right)$ не совпадает с величинами $\delta \gamma, \delta R_{ц}$ и $\delta H$. Для! исследуемого блока нмеем

$\Delta \gamma=230 \frac{\kappa 2}{\mu^{4}}, \Delta R_{ц}=83 \frac{\kappa \Gamma}{c M^{2} \mu}, \Delta H=32700 \frac{\kappa \Gamma}{c \mu^{2} \mu}$ нли $\frac{\Delta \gamma}{\bar{\gamma}}=23 \%$,

$\frac{\Delta P_{\mathrm{L}}}{\bar{R}_{\mathrm{u}}}=57 \% \quad$ и $\quad \frac{\Delta H}{\bar{H}}=63,5 \%$.

Непостоянство величин модуля упругости газокукермита в направлении заливки блока оказывает влиянне на распределение напряжений в блоке при его загружении. При кратковременном загруженин запаренного газокукермита между напряжениями и деформациями существует приб́лизительно лннейная зависимость

$$
\sigma=H \varepsilon \text {. }
$$

Предполагаем, что при загруженин поперечные сечения блока остаются плоскнми; это значнт

$$
\varepsilon=\varepsilon_{0}+\varepsilon_{1} \xi+\varepsilon_{2} \eta
$$

где $\xi=\frac{x}{a}, \eta=\frac{y}{b}-$ снстема координат (см. рнс. 1),

$\varepsilon_{0}, \quad \varepsilon_{1}, \quad \varepsilon_{2}$ - пока нензвестные параметры.

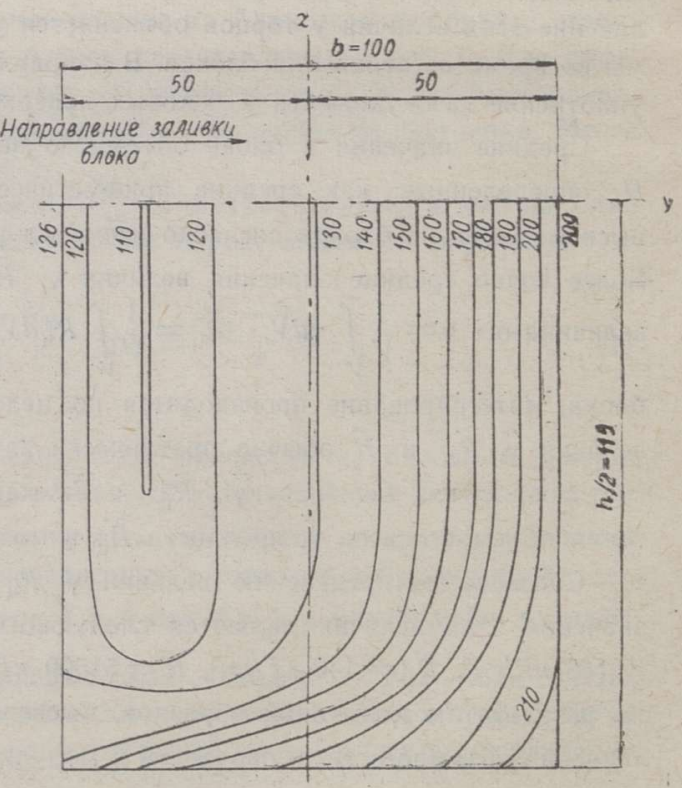

Рнс. 3. Нзменение значеннй прочности $R_{\text {II }}$ газокукермита в блоке. 
Из выражений (2) н (3) следует.

$$
\sigma=H(\xi, \eta)\left(\varepsilon_{0}+\varepsilon_{1} \xi+\varepsilon_{2} \eta\right) .
$$

Пусть внешняя нагрузка приложена в направлении продольной осн $\boldsymbol{z}$ блока с экснентрнцитетамн $e_{x}, e_{y}$ или, нспользуя безразмерные велкчины, $\omega_{\xi}=\frac{e_{x}}{a}, \omega_{\tau_{i}}=\frac{e_{y}}{b}$.

Из условий равновесия внешннх н внутренних снл следует снстема уравнений для нахождения параметров $\varepsilon_{0}, \varepsilon_{1}$ и $\varepsilon_{2}$

$$
\begin{aligned}
& I_{00} \varepsilon_{0}+I_{10} \varepsilon_{1}+I_{01} \varepsilon_{2}=\bar{\sigma}, \\
& I_{10} \varepsilon_{0}+I_{20} \varepsilon_{1}+I_{11} \varepsilon_{2}=\omega_{\xi} \bar{\sigma}, \\
& I_{01} \varepsilon_{0}+I_{11} \varepsilon_{1}+I_{02} \varepsilon_{2}=\omega_{\gamma_{i}} \bar{\sigma}
\end{aligned}
$$

где

$$
I_{m n}=\int_{-\frac{1}{2}}^{+\frac{1}{2}} \int_{-\frac{1}{2}}^{+\frac{1}{2}} \xi m \eta^{n H} H(\xi, \eta) d \xi d \eta
$$

$$
m, n=0,1,2 \text {. }
$$

$\bar{\sigma}=\frac{P}{a b}-$ среднее напряжение в болоке; $P$ - внешняя нагрузка.

Подставляя найденные из снстемы уравнений (5), величины $\varepsilon_{0}, \varepsilon_{1}, \varepsilon_{2}$ в выражение (4), находим распределение напряжений В поперечных сечениях

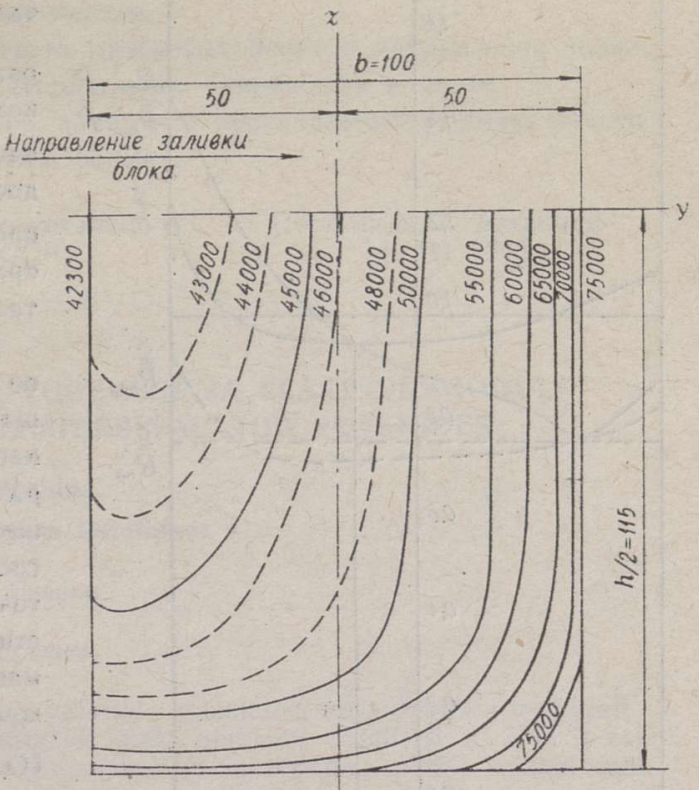

Рнс. 4. Измененне-значеннй модуля упругости $H$ газокукермита в блоке. блока.

На рис. 5 нзображена завнсимость $\frac{\sigma}{\bar{\sigma}}$ от $\eta=\frac{y}{b}$, прн $z=0$, при центральном сжатии $\left(\omega_{\xi}=\omega_{\eta}=0\right)$. Эта завнсимость найдена на основании формулы (4) с учетом значений модуля упругости, полученных по рис. 4. На том же рисунке нанесена зависимость $\frac{R_{ц}}{\bar{R}_{\mathbf{u}}}$ от $\eta$ для детально исследованного блока.

Призменная прочность материала блока $-R_{\text {nр }}$, благодаря влиянню влажности и

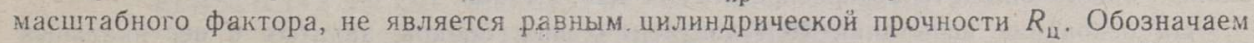
отношение призменной прочности газокукермита к цнлиндрической прочности $R_{\mathbf{u}}$ буквой $k$, тогла

$$
R_{\mathrm{np}}=k R_{\mathrm{u}}
$$

Среднее значение призменной прочности газокукермита в блоке обозначаєм символом $\bar{R}_{\text {пр }}$, которая является равным $k \bar{R}_{\text {д }}$

При блоках из однородного материала прочность блока $R_{\text {бл }}$ счнтается равной ее

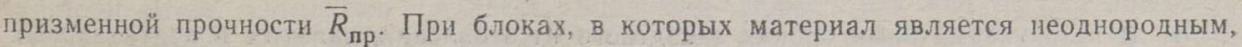
прочность блока может отличаться от средней призменной прочности $\bar{R}_{\text {пр }}$.

Так как $\bar{R}_{\text {пр }}=k \bar{R}_{\mathrm{n}}$, можем на асновании формулы (6) написать $\frac{R_{\text {пр }}}{\bar{R}_{\text {пр }}}=\frac{k R_{\mathrm{u}}}{k \bar{R}_{\mathrm{\mu}}}=\frac{R_{\mathrm{u}}}{\bar{R}_{\mathrm{\mu}}}$, следовательно, на рис. 5 вместо отношения $\frac{R_{\mathrm{\mu l}}}{\bar{R}_{\mathrm{n}}}$, можно рассматривать отношение $\frac{R_{\mathrm{np}}}{\bar{R}_{\mathrm{np}}}$. 
Из рис. 5 видно, что при достижении среднего напряжения $\bar{\sigma}$ в блоке, величины $\bar{R}_{\text {пр }}$ в части блока, где $\eta<0,12$, напряжения $\sigma$ болыше призменной прочности мате-

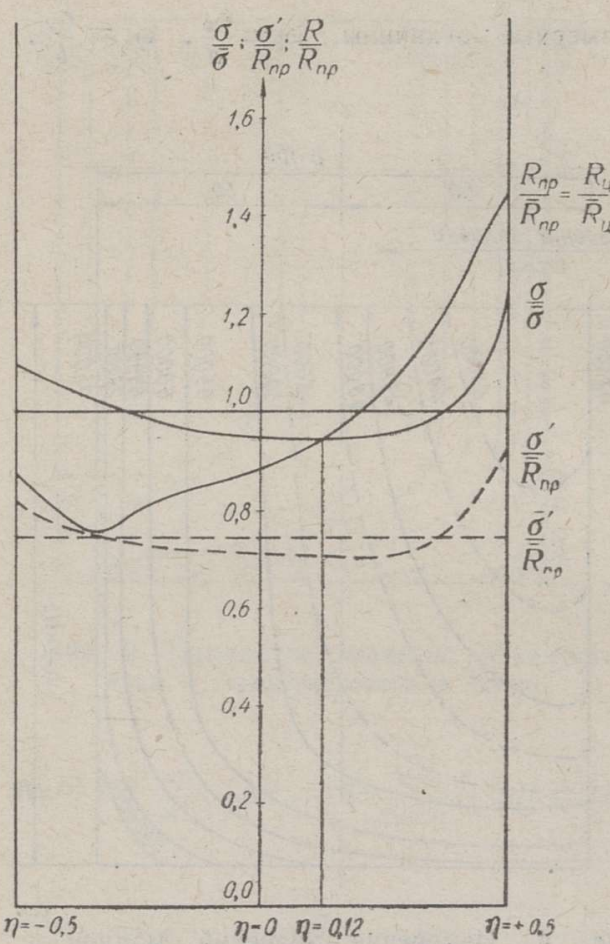

Рис. 5. Распределение напряжений $\sigma$ и прочностей $R_{\mathrm{u}}$ в поперечном сеченни блока, при $z=0$. рнала блока $R_{\text {пр }}$. так как лыния, изображающая завнсимость $\frac{R_{\text {пр }}}{\bar{R}_{\text {пр }}}$, находится в этой частн блока ниже линии $\stackrel{\sigma}{=}$. Следовательно, . . опасность разрушения зтой части блока вознккает раньше, чем среднее значение напряженик̆ $\bar{\sigma}$ при центральной нагрузке достигнет величины средней призменной прочности материала блока $\vec{R}_{\text {пр }}$, которую прн блоке кз однородного матернала считали прочностью блока $R_{\text {бл }}$.

Если в блоке сжимающие напряжения по абсолютной величнне нигде не превышают прочности. $R_{\text {пр }}=k R_{\text {щ }}$ то в таком напряженном состоянии блок, вероятно, не разрушается. Можно ожидать, что разрушение блока начинается при напряженном состоянии, когда в одной или некоторых точках блока сжимающие напряжения достигнут величины $R_{\text {np }}$. Обозначаем сжимающие напряжения в блоке в этом напряженном состоянии функцией $\sigma^{\prime}=\sigma^{\prime}(\xi, \eta)$. На рис. 5 изображена зависимость $\frac{\sigma^{\prime}}{\bar{R}_{\text {пр }}}$ от $\eta$. Среднее значение напряжений $\sigma^{\prime}$ обозначаем символом $\overline{\sigma^{\prime}}$. При эток, если распределение модулей упругостн материала нмеет вид, изображенный на рис. 4, то $\bar{\sigma}^{\prime}=0,74 \vec{R}_{\text {пр }}$. Следовательно, в конкретном случае уже при среднем значении напряжений $\bar{\sigma}^{\prime}=\frac{P^{\prime}}{a b}=0,74 \bar{R}_{\text {пр }}$ возннкает опаскость разрушения блока.

При медленном загруженни прочность блока может оказаться несколько больше вышеуказанной, так кақ перед разрушеннем возникают пластические деформации и в блоках можно ожидать перераспределения напряжений.

На основании испытания 40 газокукермнтовых блоков, залитых в вертикальнык формах, коэффициент $m=\frac{R_{6}}{\bar{R}_{\mathrm{u}}}$ имеет значение 0,40 , что примерно на $20 \%$ меньше величнны этото коэффициента для газокукермитовык блоков, залитых в горизонтальных формах. По данным Таллинского политехннческого института ${ }^{2}$, для блоков, залитых в горнзонтальных формах, коэффициент нt имеет значение прняерно 0,5 .

\section{Выводы}

1. В газокукермитовых блоках заводского изготовления, залитых в вертикальных формах, материал в блоках не является однородным. Согласно данным нсследованнй 1962 г., объемный вес и прочность блока в направлении заливки нзменнлись в среднем $186 \frac{\kappa 2}{u^{3}}$ и $64 \frac{\kappa I}{c \mu^{2}}$ на 1 погонный метр высоты залнвки.

2 См. научно-технический отчет: Gaaskukermiidist suurplokkide tugevuse kaudne määramine. Teostajad: K. Ollik, O. Roots, H. Remuna, O. Vahelaid, TPI, Tallinn 1959: a. 
2. У торцовых поверхностей блока линин постоянных значений объемного веса, прочностн и модулей упругости расположены плотнее, чем в середине блока.

3. Изменение величип объемного веса, прочности и модуля упругости в направлении заливки блоқа нелинейное; изменение этих величин по низу заливки болыше, чем по верху заливки.

4. Средние значения объемного веса, прочности и модуля упругости матернала блока можно определить на образцах, высверленных из блока по схеме, показанной на рис. 1, найдя потом их средние арифметические.

5. Изменение значения модуля упругости матернала блока в направленни залив ки оказывает существенное влияние на распределение напряжений в блоке.

6. Прочность газокукермитовых блоков, залитых в вертикальных формах, меньше прочности блоков, залитых в горизонтальных формах.

\title{
Институт строительства и строительных материалов Постунила в редакцию Государственного комитета СМ ЭССР \\ 14. III 1963
} по делам строительства

\section{GAASKUKERMIIDI MAHUKAALU, TUGEVUSE JA ELASTSUSMOODULI MUUTUMINE VERTIKAALVORMIDES VALATUD PLOKKIDES}

\author{
V. Raidna, \\ tehnikateaduste kandidaat
}

\section{H. Eesorg}

\section{Resümee}

Artiklis vaadeldakse vertikaalvormides valatud gaaskukermiitplokkide materjali tugevuse ja deformatiivsete omaduste mutumist ploki ulatuses. Joonistel 2,3 ja 4 esitatakse mahukaalu $\gamma$, tugevuse $R_{\mathrm{L}}$ ja elastsusmooduli $H$ mutumine plokis nende suuruste samaväärsusjoonte kaudu. Ploki materjali elastsusmooduli muutus plokis põhjus. tảb erineva pingete jaotuse, kui see on samal koormisel töötavas homogeensest materjalist plokis. Eeldusel, et ploki materjali puhul kehtib Hooke'i seadus ja põiklõigete tasapinnalisuse hüpotees, saab pingete jaotuse leida avalduse (4) kaudu, kus parameetrid $\varepsilon_{0}, \varepsilon_{1}$ ja $\varepsilon_{2}$ leitakse lineaarvörrandite süsteemist (5). Pingete jaotus ploki keskpunkti läbivas põiklõikes, mille puhul elastsusmoodulite jaotus vastab joonisel 4 esitatule, on toodud joonisel 5 .

\section{ALTERNATION OF SPECIFIC WEIGHT, STRENGTH AND MODULUS OF ELASTICITY IN OIL SHALE FLY ASH GAS CONCRETE BLOCKS CAST IN VERTICAL MOULDS}

\author{
V. Raidna, H. Eesorg
}

\section{Summary}

The authors present data on the alternation of properties of oil shale fly ash gas concrete in blocks cast in vertical moulds. In figs. 2,3 and 4 are given equal values lines of specific weight, strength and modulus of elasticity of gas concrete in blocks. Alternation in the values of modulus of the elasticity of gas concrete causes an alternation of stress distribution in bloch's. Supposing Hooke's law and the hypothesis of plain cross-sections be valid, the stress distributions can be found in formula (4), where parameters $\varepsilon_{0}, \varepsilon_{1}$ and $\varepsilon_{2}$ are found from the system of linear equations (5). According to the distribution of the modulus of elasticity shown in fig. 4 , the stress distribution in blocks by axial compression is given in fig. 5 .

Institute of Building and Building Materials of the State Building Committee on Construction of the Council of Ministers of the Estonian S.S.R.
Received

March 14th, 1963

5 ENSV TA Toimetised T-1 64 